\title{
Mise en évidence des instabilités d'un système d'essuyage par analyse vibratoire - Corrélation avec un modèle théorique
}

\author{
Carine Chevennement-Roux ${ }^{1}$, Jean-Pierre Lainé ${ }^{2}$, Patrick Alliot ${ }^{3}$, \\ Évelyne Aubry ${ }^{3, a}$, Thomas Dreher ${ }^{1}$ et Louis JÉzéquel ${ }^{2}$ \\ 1 Valeo Systèmes d'Essuyage, BP 581, La Verrière, 78321 Le Mesnil Saint Denis Cedex, France \\ 2 École Centrale de Lyon, 36 avenue Guy de Collongue, BP 163, 69131 Écully Cedex, France \\ 3 ESSAIM, 12 rue des frères Lumière, 68093 Mulhouse Cedex, France
}

Reçu le 20 octobre 2004, accepté le 10 mai 2006

\begin{abstract}
Résumé - Cet article présente l'étude des instabilités d'un système d'essuyage par analyse vibratoire et en propose un modèle théorique dans un but d'aide à la conception. L'optimisation d'un système d'essuyage, qui est un objectif essentiel pour un équipementier tel que Valeo, conduit le concepteur à chercher à éliminer autant que possible les différents défauts d'essuyage qui sont pour la plupart dus à l'apparition de phénomènes vibratoires entre la lame de caoutchouc et le pare-brise. Ces phénomènes apparaissent dans différentes conditions (état et humidité du pare-brise, pression de la lame de caoutchouc sur le verre, angle d'attaque du balai sur le pare-brise, galbe du pare-brise... ). Afin de limiter le nombre de paramètres intervenant dans le système, nous étudierons un système d'essuyage expérimental constitué d'un bras classique et d'une réglette rigide et plane portant une lame de caoutchouc. Nous avons donc mis en place un modèle théorique fondé sur une méthode de synthèse modale. Ce modèle nous permet de prédire les zones d'instabilités en fonction de trois paramètres qui sont apparus comme étant les plus influents à savoir : l'angle d'attaque, la force d'appui et le coefficient de frottement. La validation du modèle a consisté à confronter les zones théoriques de stabilité et d'instabilité (lieu de la bifurcation de Hopf) avec les relevés pratiques, et ceci en faisant varier les trois paramètres précités. La cohérence des résultats obtenus nous permet d'envisager une intégration du modèle dans les logiciels Métier Valeo.
\end{abstract}

Mots clés : Systèmes d'essuyage / instabilités / vibrations / modélisation / frottement / bifurcation de Hopf

\begin{abstract}
Instabilities highlighted of a wiper system by vibratory analysis - Correlation with a theoretical model. This article deals with the description of a wiper system instabilities by vibratory analysis and proposes a theoretical model of the wiper system with an aim of assistance to the design. The optimization of a wiper system, which is an essential objective for an equipment supplier such as Valeo, leads the design engineer to seek to eliminate as much as possible the various defects from wiping which for the majority are due to the appearance of vibratory phenomena between the rubber blade and the windshield. These phenomena appear under various conditions. In order to limit the number of parameter playing a role in the system, we will study an experimental wiper system consisting of a traditional arm and a flat and rigid blade holder carrying a rubber blade. Thus we proceeded to the construction of a theoretical model based on a modal synthesis method. This model allows to predict the areas of instabilities according to three parameters which seemed to be most influential such as: the attack angle, the arm forces and the friction coefficient. The validation of the model consisted in confronting the theoretical areas of stability and instability (Hopf bifurcation area) with the practical statements. The coherence of the results obtained allows to consider an integration of the model in the Valeo analytical tools set.
\end{abstract}

Key words: Wiper systems / instabilities / vibration / modelisation / friction / Hopf bifurcation

${ }^{a}$ Auteur correspondant : evelyne.aubry@uha.fr 


\section{Nomenclature}

\begin{tabular}{|ll|}
\hline$\alpha:$ & angle d'attaque (en degré) \\
$\mu:$ & coefficient de frottement \\
$F_{\mathrm{N}}:$ & force normale appliquée ou force d'appui \\
$f:$ & force de frottement \\
$V:$ & vitesse de déplacement du système \\
& d'essuyage $\left(\right.$ en $\left.\mathrm{m}^{-1}\right)$ \\
{$\left[K_{\mathrm{L}}\right]:$} & matrice raideur de la lame \\
{$[K F]:$} & matrice raideur du système complet \\
{$[M F]:$} & matrice masse du système complet \\
{$\left[U_{X}, U_{Y}, U_{Z}\right]:$} & translations selon les axes $[X, Y, Z]$ \\
{$\left[\theta_{X}, \theta_{Y}, \theta_{Z}\right]:$} & rotations selon les axes $[X, Y, Z]$ autour \\
& du point crochet \\
\hline
\end{tabular}

\section{Introduction}

Optimiser les systèmes d'essuyage afin d'éliminer les différents phénomènes d'instabilité dynamique dus au couplage entre la lame de caoutchouc et le pare-brise et générant une gêne visuelle ou auditive pour le conducteur est un objectif prioritaire. Ces instabilités apparaissant dans certaines conditions [1] : état et humidité du pare-brise, angle d'attaque du balai d'essuie-glace sur le pare-brise, galbe du pare-brise... C'est pourquoi il est intéressant de comprendre la dynamique de fonctionnement du système d'essuyage.

Le but ultime de cette étude est d'intégrer aux logiciels d'aide à la conception des systèmes d'essuyage, un outil permettant de prévoir l'apparition des instabilités (telles que le broutement ou le crissement) en fonction des différents paramètres du système. Ces logiciels Valeo permettent déjà une étude statique; notre but est alors d'y ajouter une fonctionnalité dynamique.

Dans cet article, après une brève description du système, nous proposons un modèle de simulation d'un système d'essuyage simplifié, basé sur les modèles Éléments Finis de ses sous-ensembles. L'étude expérimentale qui suit permet ensuite de mettre en évidence les phénomènes d'instabilités et de valider le modèle, dans différentes configurations.

\section{Description du système}

Un système d'essuyage (Fig. 1) est constitué classiquement d'un moteur électrique et d'un mécanisme à base de bielles et de manivelles (la timonerie) permettant de transmettre le mouvement de rotation alternatif aux bras [2]. Ceux-ci supportent les balais sur lesquels sont fixées les lames de caoutchouc qui évacuent le film d'eau sur le pare-brise [3, 4].

Plusieurs paramètres ont une influence significative sur l'apparition de défauts d'essuyage. On peut classer ces paramètres selon trois catégories : paramètres géométriques (géométrie complexe du pare-brise, zone de balayage...), paramètres physiques (raideur et distribution d'inertie du balai, comportement du frottement verre/caoutchouc...) et paramètres physico-chimiques

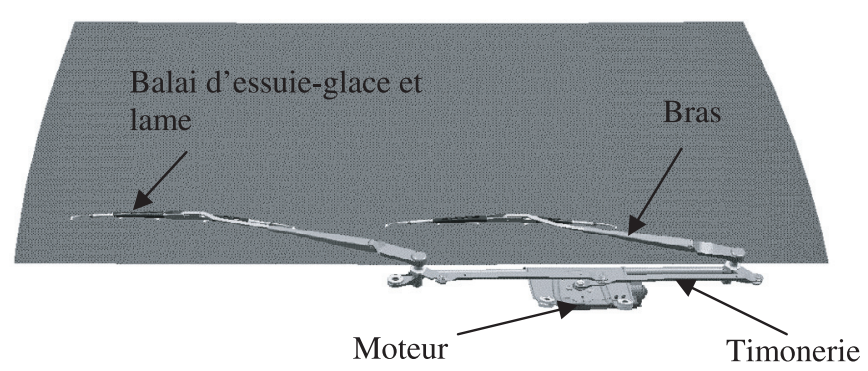

Fig. 1. Description du système d'essuyage positionné sur un pare-brise.

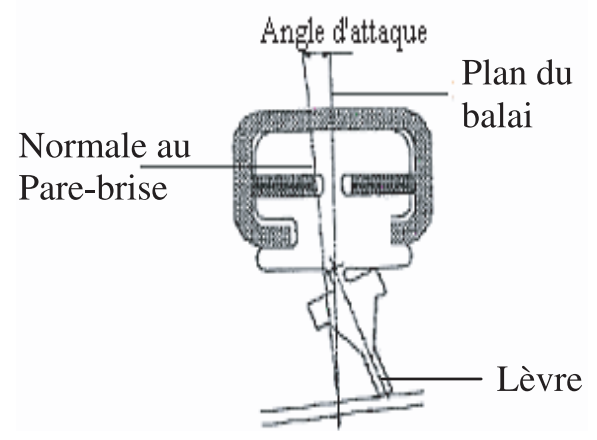

Fig. 2. Définition de l'angle d'attaque.

(nature du caoutchouc et état de propreté du verre...). Compte tenu du nombre important de paramètres du système, nous ne ferons varier que ceux qui influent le plus sur les instabilités vibratoires.

Un des paramètres géométriques prédominant pour la définition des conditions d'essuyage est l'angle d'attaque, défini comme étant l'angle entre la normale au pare-brise et le plan du balai (Fig. 2). Un autre paramètre caractérisant les conditions d'essuyage est le coefficient de frottement. En effet, celui-ci dépend de la nature du matériau et varie suivant l'état de propreté du pare-brise et l'état de surface du caoutchouc de la lame [5]. Enfin, un troisième paramètre jouant un rôle majeur sur la qualité d'essuyage est la force d'appui du bras sur le balai.

Le dispositif d'essuyage expérimental étudié est constitué d'une réglette rigide portant la lame caoutchouc, balayant une surface vitrée plane sans inversion du sens de balayage.

\section{Modèle théorique}

\subsection{Modélisation}

La modélisation numérique est basée sur le modèle Éléments Finis établi sous ANSYS du système brasréglette. Le système d'essuyage est divisé en trois sous-systèmes distincts : le bras, la réglette et enfin la lame. Nous retiendrons pour chaque sous-système les paramètres d'entrée permettant de décrire leur comportement dans le domaine des fréquences des phénomènes expérimentaux observés (par condensation, sous-structuration). 


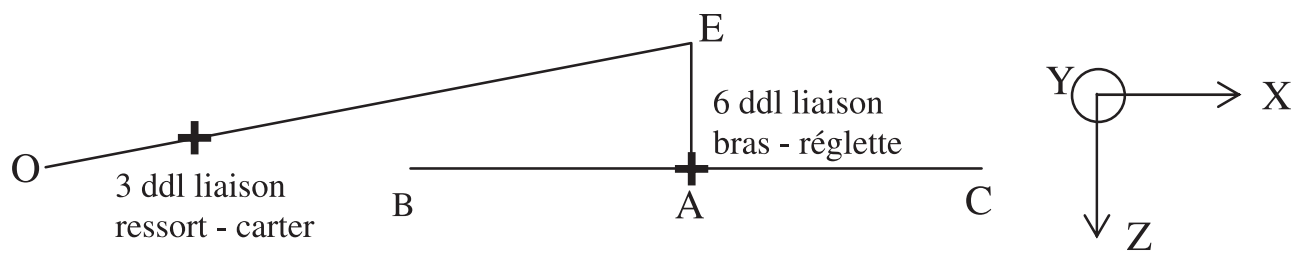

Fig. 3. Représentation schématique du système bras-réglette-lame dans le repère d'espace $(X, Y, Z)$.

La figure 3 nous donne une représentation schématique du système d'essuyage repéré selon trois axes $(X, Y, Z)$.

\subsection{Modèle du bras}

À partir du modèle Éléments Finis, nous effectuons une condensation de Guyan [6], ce qui va nous permettre d'extraire pour le bras 30 degrés de liberté maîtres. Parmi ces 30 degrés de liberté maîtres, nous en avons 3 qui sont associés au point de liaison carter-ressort de rappel et 6 qui représentent la liaison bras-réglette. Ensuite, nous extrayons les matrices de raideur et de masse du sous-système bras pour créer le modèle numérique sous Matlab.

Ayant extrait la matrice de raideur du bras, nous ajoutons la raideur du ressort de rappel au niveau du nœud de liaison carter-ressort. Puis nous appliquons sur le bras la méthode de sous-structuration de Craig et Bampton $[6,7]$ et nous obtenons un système à 8 degrés de liberté dont 6 pour la liaison bras-réglette et 2 pour les modes propres dynamiques (les deux modes de flexion, vertical $(99 \mathrm{~Hz}$ ) et tangentiel $(79 \mathrm{~Hz}))$. Nous obtenons alors pour le bras, des matrices de raideur $K$ et de masse $M$ de taille $8 \times 8$.

\subsubsection{Modèle de la réglette}

Nous considérons ensuite la réglette comme étant un corps rigide avec 6 degrés de liberté. Dans ce cas, nous n'avons pas d'énergie de déformation (matrice de raideur nulle). De plus, la liaison entre le bras et la réglette est considérée comme étant une liaison verrou autour de $Y$. Ainsi, sur les 6 degrés de liberté de la réglette, 5 sont communs avec le bras. Le sixième degré de liberté, qui est la rotation de la réglette suivant $Y$ est un degré de liberté supplémentaire pour le système complet. Le premier mode propre non nul de la réglette est à $430 \mathrm{~Hz}$; ce mode est très éloigné du domaine fréquentiel qui nous intéresse.

\subsubsection{Assemblage bras-réglette}

L'obtention des matrices de raideur et de masse finales se fait en couplant le bras et la réglette. Ainsi, la matrice de masse finale est de taille $9 \times 9$ et comprend des termes de la matrice de masse du bras et de la réglette ainsi que des termes de couplage dus à la liaison. La matrice de raideur finale est également une matrice $9 \times 9$ à laquelle il faut ajouter la matrice de raideur de la lame. Nous avons en tout neuf degrés de liberté pour le système brasréglette, soit les deux degrés de liberté dynamiques du bras, les trois translations et les trois rotations du système auxquels vient s'ajouter la rotation selon $Y$ de la réglette.

\subsubsection{Modèle de la lame}

Afin de modéliser la lame, nous avons posé différentes hypothèses :

- on néglige les forces d'inerties associées à la déformation de la lame;

- on néglige les couplages longitudinaux de la lame;

- on considère une force normale $F_{\mathrm{N}}$ appliquée au système et une force de frottement qui suit une loi de type Coulomb telle que $f=\mu F_{\mathrm{N}}[8,9]$.

Ainsi, le comportement de la lame sera défini par un modèle 2D quasi-statique (voir Sect. 3.2). Les paramètres d'entrées sont l'angle d'attaque, la force d'appui et le coefficient de frottement. Une solution non-linéaire sera obtenue en fonction de ces paramètres. Les résultats retenus seront :

- la position relative du point de contact;

- la raideur tangente autour de cette position d'équilibre $\frac{\mathrm{d} F_{\mathrm{N}}}{\mathrm{d} u z}$.

À l'aide de ces données, nous calculons le torseur des efforts au point $\mathrm{E}$ en fonction des déplacements et des rotations du point crochet. Nous obtenons la matrice de raideur de la lame $K_{\mathrm{L}}$ qui est de la forme suivante :

$$
\left[\begin{array}{l}
F_{X} \\
F_{Y} \\
F_{Z} \\
M_{X} \\
M_{Y} \\
M_{Z}
\end{array}\right]=\left[K_{\mathrm{L}}\right]\left[\begin{array}{l}
U_{X} \\
U_{Y} \\
U_{Z} \\
\theta_{X} \\
\theta_{Y} \\
\theta_{Z}
\end{array}\right]
$$

$K_{\mathrm{L}}$ est une matrice de taille $6 \times 6$, non-symétrique calculée au point E.

Notons également que nous avons tenu compte de la condition de maintien de la lame sur la vitre lors de la modélisation de celle-ci. 


\subsection{Modèle de déformation de la lame}

Il est nécessaire d'étudier le modèle théorique tout d'abord de façon statique afin de s'assurer de l'existence de solutions statiques stables. On considère la position d'équilibre statique telle que la force d'appui $F_{\mathrm{N}}$ due au ressort vienne écraser la lame sur la vitre. Ainsi, à partir de cette position, il existe trois solutions possibles pour obtenir des positions statiques stables :

- la position 1 dite dans le sens de l'essuyage;

- la position 2 en compression;

- la position 3 dite dans le sens du rebroussement.

À partir du modèle Éléments Finis de la lame, nous réalisons une étude statique de la lame. Pour des raisons de stabilité de la solution non-linéaire (solutions multiples), les calculs statiques sont réalisés en pilotant la force verticale exercée sur la réglette et le déplacement horizontal du point de contact lame-vitre. À partir des résultats de simulation, pour différentes valeurs de la force verticale appliquée sur la réglette et du coefficient de frottement lame-vitre, on peut calculer :

- La raideur verticale tangente définie par la dérivée $\frac{\mathrm{d} F_{\mathrm{N}}}{\mathrm{d} u z}$ à $\mu$ constant autour de la position d'équilibre.

- Le déplacement horizontal du point de contact par rapport à la réglette : uy

- Le déplacement vertical de la réglette par rapport à la vitre : uz.

Ces déplacements sont exprimés par rapport à la configuration de référence suivante : lame non-déformée en contact avec la vitre.

- Les solutions de type 1 correspondent à une situation d'essuyage avec $V>0$.

- Les solutions de type 2 pour lesquelles nous avons $\mu>0$ correspondent soit à une situation d'essuyage lorsque $V>0$ pour $u y<0$, soit à une situation de rebroussement lorsque $V>0$ pour $u y>0$.

- Les solutions de type 3 pour lesquelles nous avons $\mu>$ 0 correspondent à une situation de rebroussement avec $V>0$.

Ainsi, le résultat du calcul statique va nous permettre d'obtenir le déplacement horizontal uy, le déplacement vertical $u z$. Ceci nous donnera alors la valeur des paramètres $a$ et $b$ tels que $a=a_{0}+u y$ où $a_{0}$ correspond à la distance horizontale entre le point de contact de la lame avec la vitre sans déformation et le point crochet et $u y$ est le déplacement horizontal du point de contact par rapport à cette position de référence $a_{0}$. De la même façon, nous avons $b=b_{0}+u z$ pour le déplacement vertical.

La raideur verticale $k$, sera calculée par double interpolation des résultats de simulations statiques. En effet, afin d'obtenir une meilleure précision du calcul de $k$, nous effectuons une première interpolation en fonction de la force appliquée puis une deuxième en fonction du coefficient de frottement. Notons que pour chaque type de solution, il existe des solutions statiques potentiellement stables et des solutions statiques potentiellement instables suivant le signe de $k$. Ainsi, pour les solutions de type 1 nous avons $k>0$ et donc des solutions statiquement stables; il en est de même pour les solutions de type 3 . En ce qui concerne les solutions de type $2, k$ peut être négatif ou positif; ce qui donne des solutions de type 2 stables ou instables en statique. Les paramètres $a, b$ et $k$ sont caractéristiques de chaque lame et constituent les paramètres d'entrées pour l'étude dynamique.

\section{4 Étude des instabilités de flottement}

\subsubsection{Zones d'instabilités}

Après avoir calculé les matrices de raideur et de masse correspondant au système bras + réglette + lame, nous aboutissons à un système de la forme :

$$
[M F][\ddot{Q}]+[K F][Q]=0
$$

avec $Q$ le vecteur généralisé tel que :

$$
Q^{T}=\left[d 1 d 2 U_{X} U_{Y} U_{Z} \theta_{X} \theta_{Y b} \theta_{Z} \theta_{Y r}\right]
$$

où $d 1$ et $d 2$ représentent les deux premiers modes propres dynamiques du bras et $U_{X}, U_{Y}$ et $U_{Z}$ représentent les translations et $\theta_{X}, \theta_{Y}, \theta_{Z}$ représentent les rotations dans les trois directions du point crochet. Précisons que $\theta_{Y b}$ correspond à la rotation suivant $Y$ du bras et que $\theta_{Y r}$ correspond à la rotation suivant $Y$ de la réglette. De plus $[M F]$ correspond à la matrice de masse finale de l'ensemble du système et $[K F]$ correspond à la matrice de raideur finale du système.

Pour chaque type de solution dans les zones où il existe un point d'équilibre statique stable, nous allons étudier leur stabilité dynamique. Nous allons alors réaliser une étude paramétrique en fonction des deux paramètres d'entrée, la force d'appui $F_{\mathrm{N}}$ et le coefficient de frottement $\mu$, pour plusieurs valeurs d'angles d'attaques.

Pour réaliser cette étude dynamique, nous allons calculer les vecteurs propres et les valeurs propres du système d'équation (Éq. (2)) pour détecter les points de flottement (valeurs propres à partie réelle positive et à partie imaginaire non nulle) [10].

Observons les domaines d'instabilité pour les solutions de type 1 avec un angle d'attaque de $15^{\circ}$, lorsqu'on fait varier la force d'appui de $10 \mathrm{~N} . \mathrm{m}^{-1}$ à $30 \mathrm{~N} . \mathrm{m}^{-1}$ et le coefficient de frottement $\mu$ de 0 à 1,2 . La figure 5 donne un exemple des zones d'instabilités dans le plan $\left(F_{\mathrm{N}}, \mu\right)$.

En comparant les différents graphes obtenus pour différents angles d'attaque, nous pouvons observer que pour des angles d'attaque supérieurs à $9^{\circ}$, il apparaît des zones d'instabilité alors que pour des angles d'attaques inférieurs à $9^{\circ}$, le système est toujours stable (réf. Sect. 5). L'angle d'attaque a donc une grande influence sur la présence d'instabilités. 


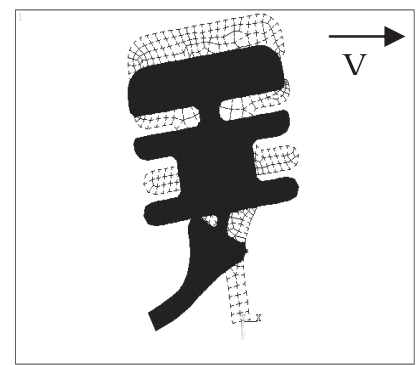

Solutions 1 : essuyage

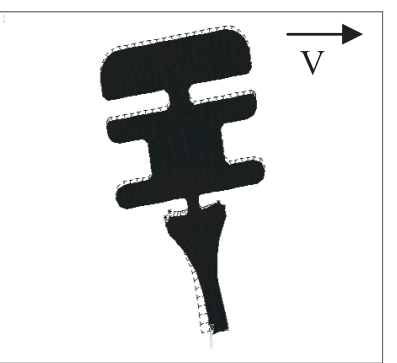

Solutions 2 : compression

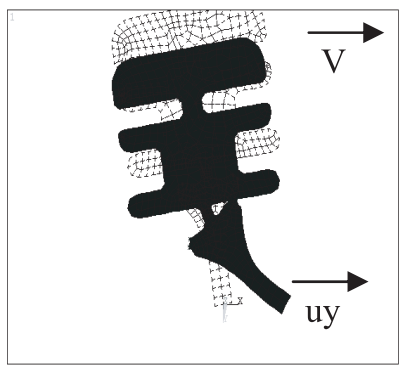

Solutions 3 : rebroussement

Fig. 4. Positions de la lame.
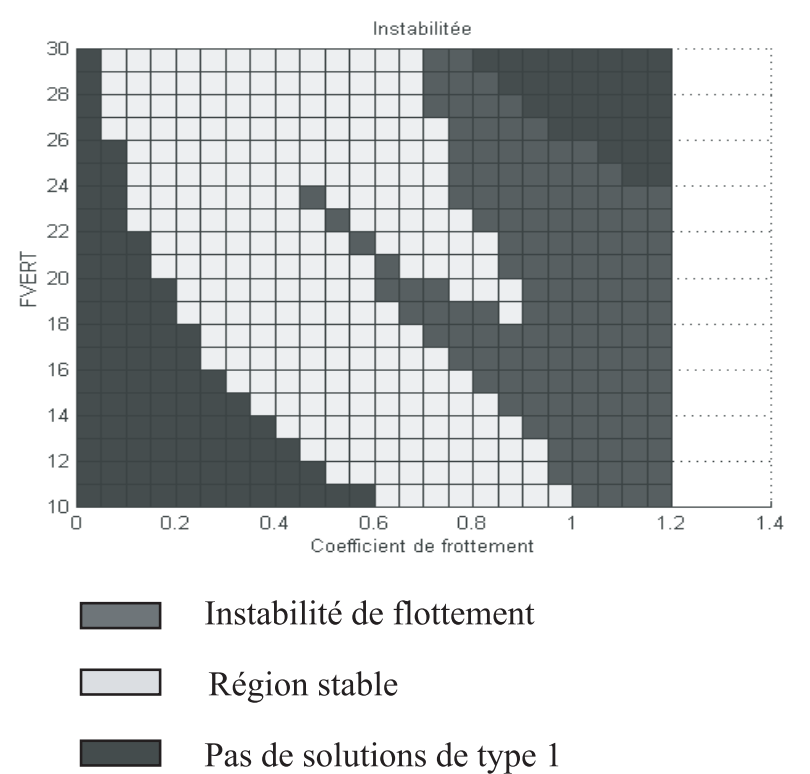

Instabilité de flottement

Région stable

Pas de solutions de type 1

Fig. 5. Régions d'instabilité pour les solutions de type 1 pour un angle d'attaque de $15^{\circ}$.

\subsection{2 Évolution des pôles}

Pour étudier la stabilité du système bras-réglette, il est intéressant de visualiser l'évolution des pôles du système en fonction du coefficient de frottement $\mu$, ceci pour un angle d'attaque fixé et une force d'appui donnée. Nous allons alors nous intéresser aux parties réelles et aux parties imaginaires des solutions de l'équation du mouvement (Éq. (2)).

Prenons alors un exemple où l'angle d'attaque vaut $15^{\circ}$ avec une force verticale de $15 \mathrm{~N} . \mathrm{m}^{-1}$, ceci pour une variation du coefficient de frottement $\mu$ de 0 à 1,2 (Fig. 6).

Sur la figure 6 , nous pouvons distinguer deux zones :

- une première zone où $0<\mu<0,8$, où il apparaît des pôles à parties réelles nulles; ceci correspond alors à un domaine stable;

- une deuxième zone où $\mu>0,8$, où il apparaît des pôles à parties réelles positives et à parties imaginaires non-nulles; ceci correspond alors à une zone d'instabilité de flottement. En effet, dans cette zone instable, nous avons un point de bifurcation pour $\mu=0,8$ avec coalescence de modes $[11,12]$; ainsi il y a couplage des modes 2 et 3 ce qui donne lieu à une instabilité.

Dans ce cas, l'instabilité de flottement apparaît pour un angle $\mu>0,8$ avec une fréquence de $25 \mathrm{~Hz}$.

\subsection{3 Étude de la déformée des modes propres}

Le calcul des modes propres des matrices de masse et de raideur va nous permettre de détecter les fréquences propres des premiers modes d'instabilité. L'évolution des pôles, nous montre que les modes qui créent l'instabilité sont les modes à $28 \mathrm{~Hz}$ et à $30 \mathrm{~Hz}$ qui correspondent aux modes 2 et 3 . Ces modes sont associés à des déplacements selon $Z$ du système bras-réglette (on observe des mouvements de «pompage » du bras et de «tangage » de la réglette). Ainsi les modes instables correspondent à un mouvement selon $U_{Y}$ pour le bras couplé à un mouvement selon $U_{z}$ pour la réglette.

En affinant le modèle pour un type particulier de lame, il est alors possible de réaliser une étude paramétrique pour différents angles d'attaque en fonction de la force d'appui et du coefficient de frottement. Cette étude nous permet alors de définir les zones stables dynamiquement et les zones instables dynamiquement pour un type de lame donné. Nous avons alors pu constater que force d'appui et coefficient de frottement sont deux paramètres qui ont une grande influence sur les phénomènes d'instabilités, ainsi que l'angle d'attaque.

\section{Analyse expérimentale des instabilités}

\subsection{Dispositif expérimental}

Plusieurs essais, sous différents angles d'attaques et différentes forces d'appui, ont été réalisés. La mise en place d'accéléromètres sur la réglette permet de quantifier les niveaux vibratoires. On utilise la vitre plane avec la réglette métallique et le bras droit. Les conditions d'essais sont glace mouillée, glace séchante et petite vitesse.

La réglette est instrumentée avec deux accéléromètres triaxiaux placés à chaque extrémité de la réglette ainsi qu'un troisième accéléromètre triaxial placé vers le centre de la réglette. Les positions de ces trois accéléromètres 

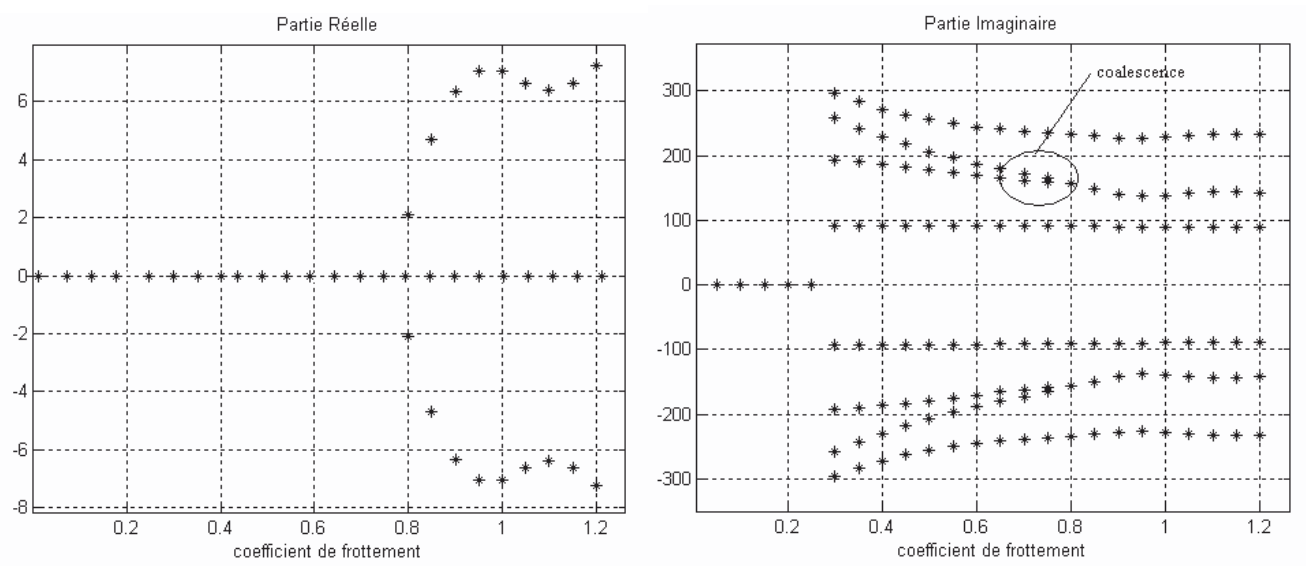

Fig. 6. Comportement des pôles du système pour $F=15 \mathrm{~N} \cdot \mathrm{m}^{-1}$ pour un angle d'attaque de $15^{\circ}$.
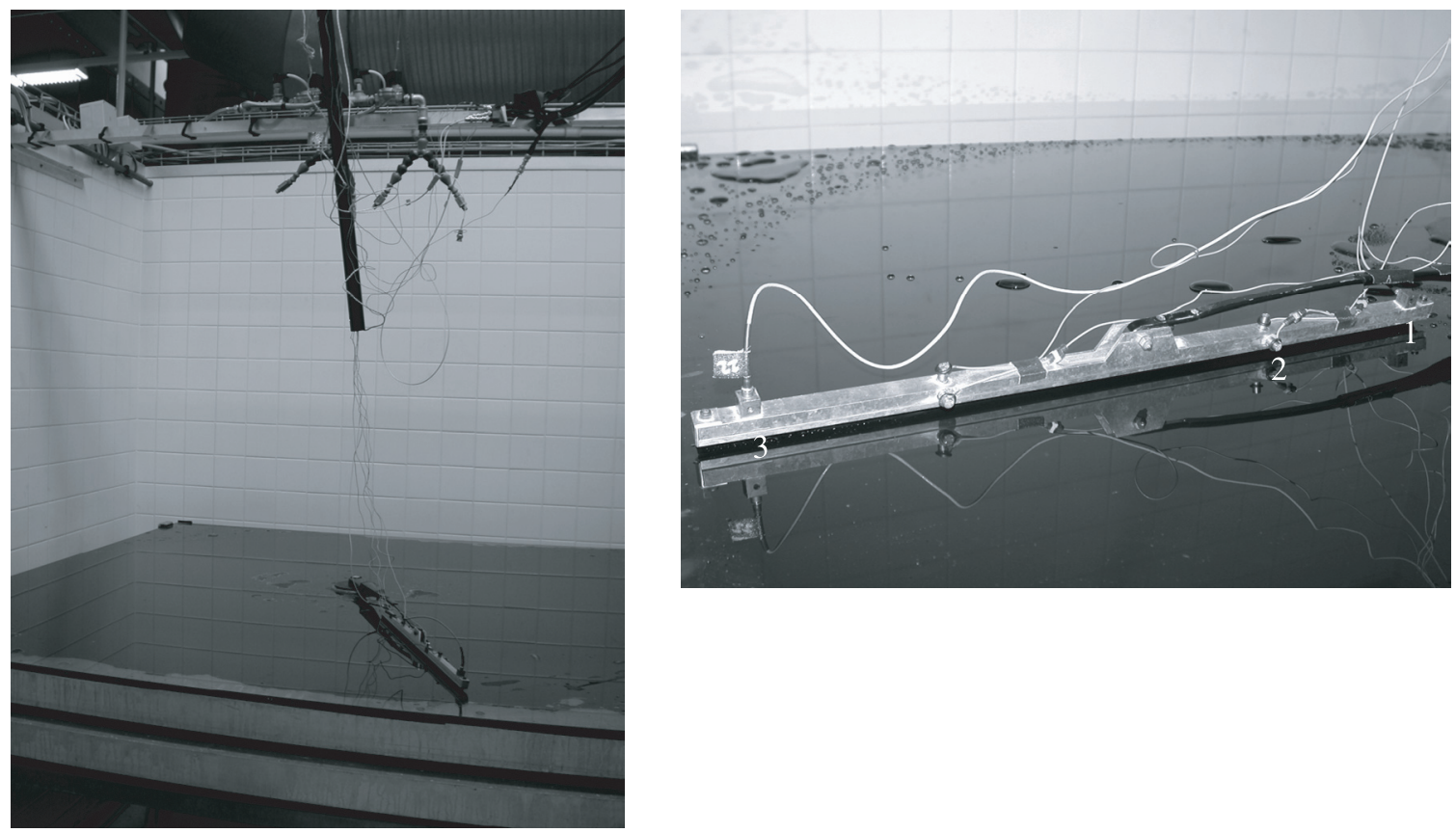

Fig. 7. Dispositif expérimental.

correspondent aux positions 1,2 et 3 comme indiqué sur la photo de la figure 7 .

Précisons que nous nous intéressons aux déplacements dans le plan $Y Z$, où la direction $Z$ est la direction perpendiculaire à la vitre et la direction $Y$ est la direction du déplacement de la lame.

On réalise une étude paramétrique afin d'obtenir un domaine d'instabilité en broutement en faisant varier deux paramètres tels que la pression $(10,12,15,17$ et 20 N.m $\left.{ }^{-1}\right)$ et l'angle d'attaque $\left(9^{\circ}, 12^{\circ}\right.$ et $\left.15^{\circ}\right)$.

\subsection{Résultats et observations}

Les figures 8 et 9 présentent un exemple de spectres d'amplitude obtenus. En observant ces deux exemples de graphes, nous pouvons tout d'abord constater que lorsque les instabilités de broutement apparaissent, le niveau des amplitudes est 100 fois plus élevé que lorsque le système est stable. De plus, nous remarquons l'existence d'un premier mode à $22 \mathrm{~Hz}$, puis d'un mode à $77 \mathrm{~Hz}$ selon la direction $z$ et qui n'apparaît pas dans la direction $y$ et enfin, un mode à $210 \mathrm{~Hz}$. On peut également remarquer que lorsque le broutement apparaît, le pic à $22 \mathrm{~Hz}$ est prédominant. En comparant les différents graphes, nous constatons que les amplitudes obtenues par l'accéléromètre 1 sont toujours plus élevées que les amplitudes issues de l'accéléromètre 3 placé à l'autre extrémité de la réglette.

D’une façon générale, nous pouvons noter la présence d'instabilités pour un angle d'attaque de $15^{\circ}$ où les niveaux sont plus élevés en glace séchante qu'en glace mouillée, ces instabilités s'atténuent pour un angle de $12^{\circ}$, et enfin nous n'observons plus d'instabilités pour un angle 

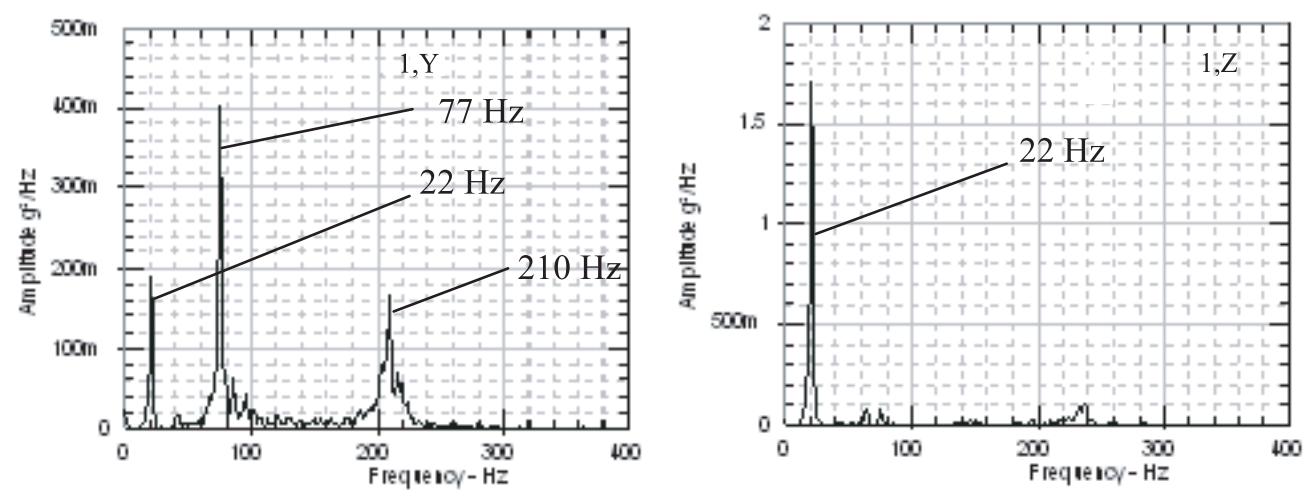

Fig. 8. Mesures vibratoires selon $Y$ et selon $Z$ dans les conditions expérimentales suivantes : glace sèche, angle d'attaque de $15^{\circ}$ et pression de $7 \mathrm{~N} \cdot \mathrm{m}^{-1}$ sans broutement.
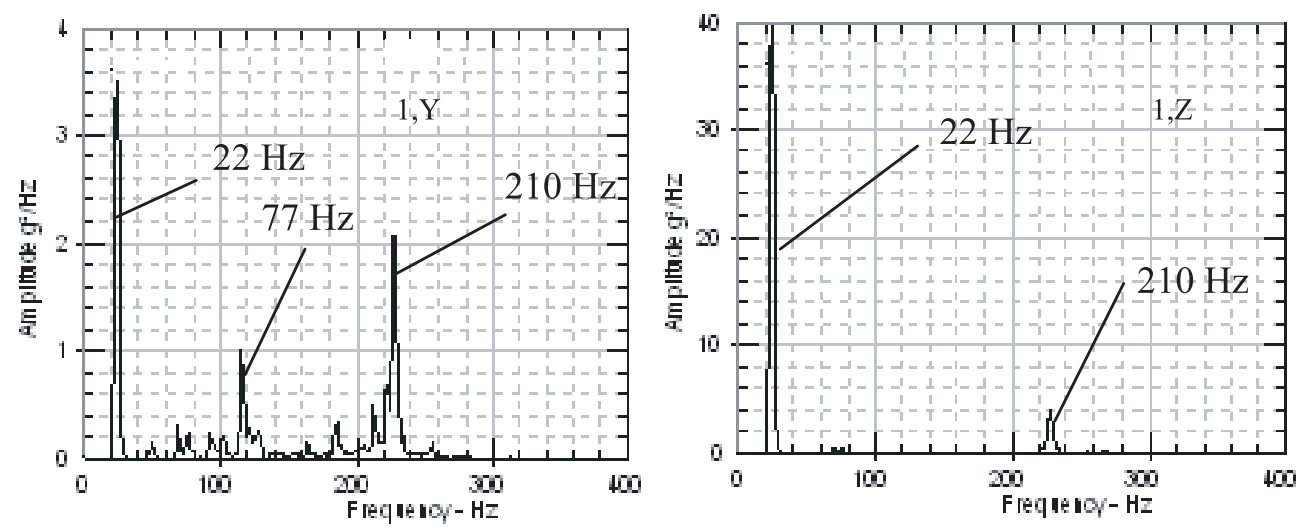

Fig. 9. Mesures vibratoires selon $Y$ et selon $Z$ dans les conditions expérimentales suivantes : glace sèche, angle d'attaque de $15^{\circ}$ et pression de $15 \mathrm{~N} . \mathrm{m}^{-1}$ avec broutement.

d'attaque de $9^{\circ}$ où les niveaux des signaux sont faibles et de même amplitude en glace mouillée et en glace séchante. Pour les angles d'attaque de $15^{\circ}$ et $12^{\circ}$, où ont lieu les instabilités, nous remarquons que les amplitudes des spectres sont plus importantes pour des forces d'appui plus faibles.

\subsection{Positions d'équilibres expérimentales}

À l'aide d'une caméra rapide, nous avons réalisé des films de lame d'essuie-glace, ceci afin d'observer le comportement de la lame lors des instabilités de broutement. Différentes configurations ont été étudiées et filmées afin d'identifier le comportement et la position que prend la lame lors de son déplacement avec et sans broutement.

\subsubsection{Position d'essuyage}

Dans un premier temps, nous avons enregistré le comportement de la lame lorsque la lèvre est orientée inverse au déplacement. L'angle d'attaque étant réglé à $12^{\circ}$ et la force d'appui à $13 \mathrm{~N} . \mathrm{m}^{-1}$, nous enregistrons les films de la lame se déplaçant dans le sens de l'essuyage. Avec une telle configuration, le broutement n'apparaît pas.
Nous effectuons ces films en condition de petite vitesse (43 tr.min ${ }^{-1}$ ), glace sèche et glace mouillée. En observant ces films nous pouvons constater que la lame reste toujours calée du même côté sans aucune instabilité, ce qui facilite le déplacement dans le sens de l'essuyage. Nous avons pu remarquer le même phénomène à grande vitesse

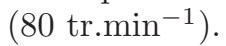

\subsubsection{Position de rebroussement}

Afin que le broutement soit visible et puisse être filmé par la caméra rapide, nous avons augmenté la force d'appui à $18 \mathrm{~N} \cdot \mathrm{m}^{-1}$ et l'angle d'attaque à $16^{\circ}$. Ainsi, nous avons enregistré les films de la lame en condition de broutement; la lame se déplaçant alors dans le sens du rebroussement. Ces films ont été réalisés en petite vitesse et en glace séchante.

Ainsi, lorsque le broutement apparaît, nous pouvons constater que la lame passe d'une position calée à une position verticale pour revenir à sa position calée. Ce mouvement de lame est alors répété pendant toute la durée du broutement. Nous retrouvons ce phénomène aussi bien en petite vitesse qu'en grande vitesse. 
Tableau 1. Exemples de conditions d'obtention du phénomène de broutement.

\begin{tabular}{|c|c|c|}
\hline Coefficient de frottement & $0,2-0,4$ & $>1$ \\
Angle d'attaque/Effort & & \\
\hline $9^{\circ}$ et $10 \mathrm{~N} \cdot \mathrm{m}^{-1}$ & Pas d'instabilité & Pas d'instabilité \\
\hline $9^{\circ}$ et $15 \mathrm{~N} \cdot \mathrm{m}^{-1}$ & Pas d'instabilité & Pas d'instabilité \\
\hline $9^{\circ}$ et $20 \mathrm{~N} \cdot \mathrm{m}^{-1}$ & Pas d'instabilité & Pas d'instabilité \\
\hline
\end{tabular}

\begin{tabular}{|c|c|c|}
\hline Coefficient de frottement & $0,2-0,4$ & $>1$ \\
Angle d'attaque/Effort & & \\
\hline $15^{\circ}$ et $10 \mathrm{~N} \cdot \mathrm{m}^{-1}$ & broutement & broutement \\
\hline $15^{\circ}$ et $15 \mathrm{~N} \cdot \mathrm{m}^{-1}$ & petit broutement & broutement \\
\hline $15^{\circ}$ et $20 \mathrm{~N} \cdot \mathrm{m}^{-1}$ & petit broutement & broutement \\
\hline
\end{tabular}
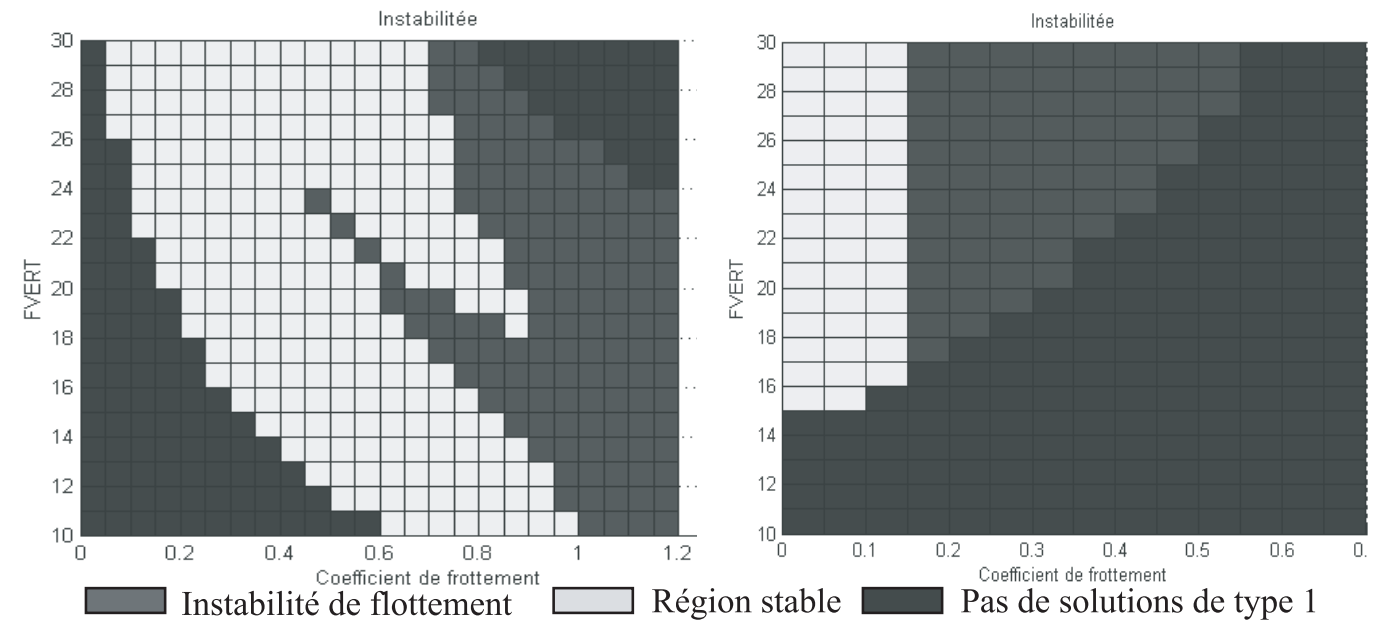

Fig. 10. Régions d'instabilité pour les solutions de type essuyage pour un angle d'attaque de $15^{\circ}$ - à gauche et pour des solutions de type rebroussement - à droite.

\subsection{Influence du coefficient de frottement sur l'apparition des instabilités}

Le but de ces mesures est de relever le coefficient de frottement obtenu lors des essais de broutement sur vitre mouillée et vitre séchante. Une étude paramétrique est réalisée. En effet, on fait varier l'angle d'attaque ainsi que la force appliquée. De plus, le sens de déplacement de la lame est celui du rebroussement pour ainsi pouvoir favoriser et étudier le broutement. Nous allons alors relever les informations données par le couplemètre situé sous l'axe du moteur.

Ces essais ont été réalisés avec les variations de paramètres suivantes:

Pression $=10,12,15,17,20{\mathrm{~N} . \mathrm{m}^{-1}}^{-1}$ et Angle Attaque $=9^{\circ}, 12^{\circ}, 15^{\circ}$.

Le tableau 1 présente quelques valeurs obtenues lors des essais. Précisons, qu'en vitre mouillée, le coefficient de frottement varie entre 0,2 et 0,4 . En vitre sèche, il varie entre 0,8 et 1 et en glace séchante, il est supérieur à 1 .

\section{Validation du modèle théorique}

La validation du modèle théorique est basée sur la confrontation entre les résultats issus de ce modèle et les résultats des essais. C'est notamment à travers les comparaisons des zones de stabilité et d'instabilité théoriques et expérimentales que nous pouvons estimer la qualité du modèle. En effet, pour le concepteur d'un système d'essuyage, c'est la prévision des conditions paramétriques d'instabilité qui importe. Nous pouvons observer les résultats de simulation sur les figures 10 et 11 .

Expérimentalement, pour un angle de $15^{\circ}$ et des forces d'appui de 10,15 et 20 N.m ${ }^{-1}$, le système est toujours instable pour $\mu>1$, et nous relevons quelques petites instabilités pour $0,2<\mu<0,4$. Nous retrouvons ces résultats sur les graphes théoriques.

Expérimentalement, pour un angle de $9^{\circ}$ et des forces d'appui de 10, 15 et $20 \mathrm{~N} . \mathrm{m}^{-1}$, le système est toujours stable pour $0,2<\mu<0,4$ et pour $\mu>1$. Nous retrouvons également ces résultats sur le graphe théorique. Nous pouvons constater qu'il y a corrélation entre les résultats expérimentaux et théoriques. Ainsi, le modèle développé permet une bonne appréhension du phénomène d'instabilité. 

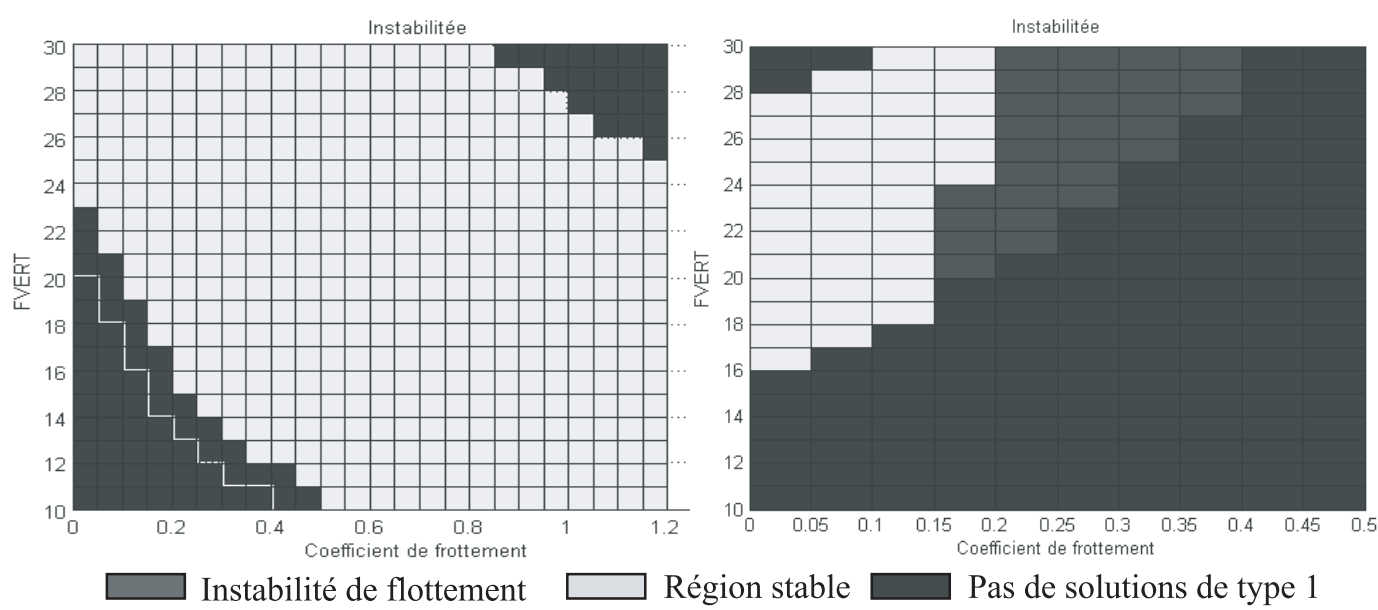

Fig. 11. Régions d'instabilité pour les solutions de type essuyage pour un angle d'attaque de $9^{\circ}$ - à gauche et pour des solutions de type rebroussement - à droite.

\section{Conclusion}

À ce stade du projet, nous pouvons avancer les premières conclusions et en tirer des perspectives d'avenir. Le modèle développé permet une bonne approche prédictive des phénomènes d'instabilités sur un système d'essuyage expérimental. La phase de validation a donné des résultats très satisfaisants en termes de corrélation avec le système réel. L'étude paramétrique classique semble suffisante et plus facile à mettre en œuvre qu'un plan d'expérience compte tenu de la difficulté de mesure, d'obtention et de reproductibilité des conditions de frottement.

Le modèle doit ensuite être adapté à la nouvelle génération de balais (les Valeo Flat Blade). Ceci impose de prendre en compte un balai très déformable et précontraint par la force au crochet. Il s'en suivra une nouvelle étape de validation par campagne d'essais.

L'intégration du modèle en tant qu'outil de simulation dans les logiciels Métier de Valeo Système d'Essuyage constituera l'ultime étape de ce projet.

\section{Références}

[1] M. Begout, Les problèmes liés au frottement élastomère - verre dans l'automobile, Mémoire de thèse, université Paul Sabatier Toulouse, $\mathrm{n}^{\circ} 2206,1979$

[2] S. Okura, T. Sekigushi, Dynamic analysis of blade reversal behaviour in a windshield wiper system, SAE Papers, 2000-01-0127, 2000
[3] V. Codfert, Modélisation globale d'un système d'essuyage, Mémoire de thèse, université des Sciences et Technologies de Lille, 1997

[4] R. Grenouillat, Étude des défauts d'essuyage : application à l'analyse prédictive, Mémoire de thèse, École Centrale de Lyon, 2001

[5] A. Koenen, Rapport de synthèse sur le frottement du caoutchouc, Rapport $n^{\circ} 0398$ 186, Laboratoire de Tribologie VSE, La Verrière, 1998

[6] J.F. Imbert, Analyse des structures par éléments finis, Cepadues Éditions, ISBN 2.85.428.273.6, 1995

[7] R.R. Craig, M.C.C. Bampton, Coupling of substructures for dynamic analysis, Am. Inst. Aeronautics \& Astronautics J. 6 (1968) 1313-1319

[8] P. Chambrette, Stabilité des systèmes dynamiques avec frottement sec : application au crissement des freins à disque, Mémoire de thèse, École Centrale Lyon, n $91-48$, 1990

[9] J.P. Boudot, Modélisation des bruits de freinage des véhicules industriels, Mémoire de thèse, École Centrale Lyon, $n^{\circ}$ 95-08, 1995

[10] J.J. Sinou, Synthèse non-linéaire des systèmes vibrants. Application aux systèmes de freinage, Mémoire de thèse, École Centrale de Lyon, 2002

[11] J. Guckenheimer, P. Holmes, Nonlinear Oscillations, Dynamical Systems, and Bifurcations of vector Fields, Springer-Verlag, 1986

[12] A.H. Nayfeh, B. Balachandran, Applied Nonlinear Dynamics: Analytical, Comptational and Experimental Methods, John Wiley \& Sons, 1995 